\title{
un calcímetro gravimétrico
}

\author{
J. CALLEJA CARRETE \\ Doctor en Ciencias
}

En el control de materias primas (calizas * y margas) y de crudos para la fabricación de cemento portland, es frecuente, como operación de rutina continuamente practicada, la determinación del contenido de carbonato cálcico. En realidad, lo que se determina es la riqueza en carbonatos totales (cálcico y magnésico), expresada globalmente como si se tratase sólo de carbonato cálcico.

Para ello se suelen utilizar métodos volumétricos fundados, bien en una valoración alcalimétrica de retorno, para determinar la cantidad de ácido que queda sin gastar, después de atacar en caliente una muestra conocida con un exceso del mismo, - bien en una medida del volumen de $\mathrm{CO}_{2}$ desprendido en el ataque total de la muestra, recogido en las debidas condiciones.

El primer método, como más sencillo, es el utilizado corrientemente en los laboratorios de las fábricas de cemento. El segundo, más delicado, pero también más exacto, se emplea todavía aunque bastante menos, $y$, sobre todo, sirve muchas veces como control o patrón de otros métodos. En él se hace uso del aparato conocido en la industria del cemento con el nombre de. kcalcímetro». Suele tener éste diversas disposiciones prácticos (1) pero, en esencia, consiste en un recipiente donde se verifica el ataque ácido de la muestra, conectado en forma estanca con una bureta de tipo Hempel para gases. El resultado de la medida del $\mathrm{CO}_{2}$ recogido, hecha a la presión atmosférica y a la temperatura ambiente, debe ser corregido y expresado en condiciones normales. Ello exige disponer de un barómetro de cierta precisión. También se impone una corrección debida a la solubilidad del anhidrido carbónico en el líquido de la bureta, salvo que este líquido se sature de dicho gas inmediatamente antes de la determinación, y en las condiciones de presión y temperatura en que ha de hacerse la lectura del volumen de $\mathrm{CO}_{2}$ recogido.

Por todas estas razones, el empleo del cọicímetro ha quedado un tanto relegado.

Las determinaciones de anhídrido carbónico son, pues, delicadas, lentas y poco cómodas, siempre que se basan en el desprendimiento del gas y en la absorción o recogida del volumen del mismo. Los métodos de absorción, tales como el que, por ejemplo, se viene empleando en la determinación del $\mathrm{CO}_{2}$ en las cales y calizas (2) y también en los yesos (3), pueden calificarse de gravimétricos, en contraposición con los que utilizan algún tipo de calcímetro (1)(4) (5) (6), los cuales son netamente volumétricos.

En la Sección de Análisis y Ensayos del I. T. C. C. se ha tratado de poner a punto un sistema de determinación de $\mathrm{CO}_{2}$ en calizas, margas y crudos para cemento, que, pudiendo tener la precisión de los métodos calcimétricos, carezca de muchos de sus inconvenientes, fales como las obligadas correcciones, para lo cual se trabaja en él por gravimetría, en lugar de hacerlo por volumetría de gases. Se trata también de simplificar al móximo el equipo necesario, evitando los complicados y frógiles montajes que a veces se hacen para la determinación del anhídrido carbónico. De aquí, el título de este trabajo.

El método consiste en expulsar, mediante el ataque ácido de una muestra del ma- 
terial, combinado con un arrastre gaseoso, todo el $\mathrm{CO}_{2}$ desprendido en dicho ataque y contenido en aquélla. La eficacia del procedimiento estriba en que todo el anhídrido carbónico se elimine y nada que no sea $\mathrm{CO}_{2}$ (humedad, por ejemplo) pueda escapar. De este modo no es preciso absorber el gas desprendido ni medir su volumen, sino que basta con hallar una diferencia de dos pesadas para tener el peso de $\mathrm{CO}_{2}$ correspondiente a la muestra analizada. Naturalmente, el procedimiento no es apropiado cuando el material analizado contiene sustancias que por ataque ácido pueden dar lugar al desprendimiento de materias volátiles distintas del anhídrido carbónico (sulfuros, etc.). Sin embargo, esto no es frecuente cuando se trata de crudos o materias primas para fabricar cemento.

\section{APARATO}

En su conjunto está representado en las fotografías de las figuras 1 y 2, y consta, en esencia, de dos tubos de absorción $T_{1}$ y $\mathrm{T}_{\mathrm{g}}$ para la retención de la humedad del aire de arrastre y de la atmósfera exterior, respectivamente, y del pequeño matraz $E$, con sus accesorios. El detalle de este último se aprecia en el esquema de la figura 3 .

Va provisto de dos tubos angulares con sendas llaves de paso $A_{1}$ y $A_{2}$ y ajustes esmerilados intercambiables que se adaptan a las embocaduras situadas en la parte superior de los cuerpos $B_{1}$ y $B_{2}$. Estos van soldados al bulbo del matraz E. El $B_{1}$ a través de la llave de paso $C$ se prolonga hacia el interior del matraz por medio de un tubo estrecho $y$ curvado $D$ que queda muy próximo al fondo.

El cuerpo $\mathrm{B}_{2}$ contiene en su interior una campana de vidrio $\mathrm{G}$, dentro de la cual va un tubo I, abierto en sus extremos y provisto de unos ensanchamientos en forma de bolas, que es el que establece la comunicación entre el bulbo del matraz y la parte superior de la campana $G$. El espacio comprendido entre ésta y el tubo I comunica con el comprendido entre la misma y la pared externa del cuerpo $B_{2}$ por el orificio $H$ practicado en la parte inferior de la campana $G$.
Por último, el bulbo del matraz va provisto de una boca $F$ con cierre de tapón esmerilado.

\section{PROCEDIMIENTO}

El matraz con sus accesorios se lava, enjuga y seca interiormente mediante una corriente de aire caliente y seco $y$, finalmente, se deseca en una estufa a $105-110^{\circ} \mathrm{C}$ durante el tiempo preciso y se deja enfriar en un desecador. Una vez frío y con la llave $C$ cerrada, se pone en el interior del cuerpo $\mathrm{B}_{1}$ ácido clorhídrico de concentración (1:4) $\mathrm{y}$ en el cuerpo $\mathrm{B}_{2}$ ácido sulfúrico concentrado, en cantidad que aproximadamente ocupe de un tercio a un medio de la altura total del tubo I. Se colocan los tubos ${ }_{\infty}^{*}$ con

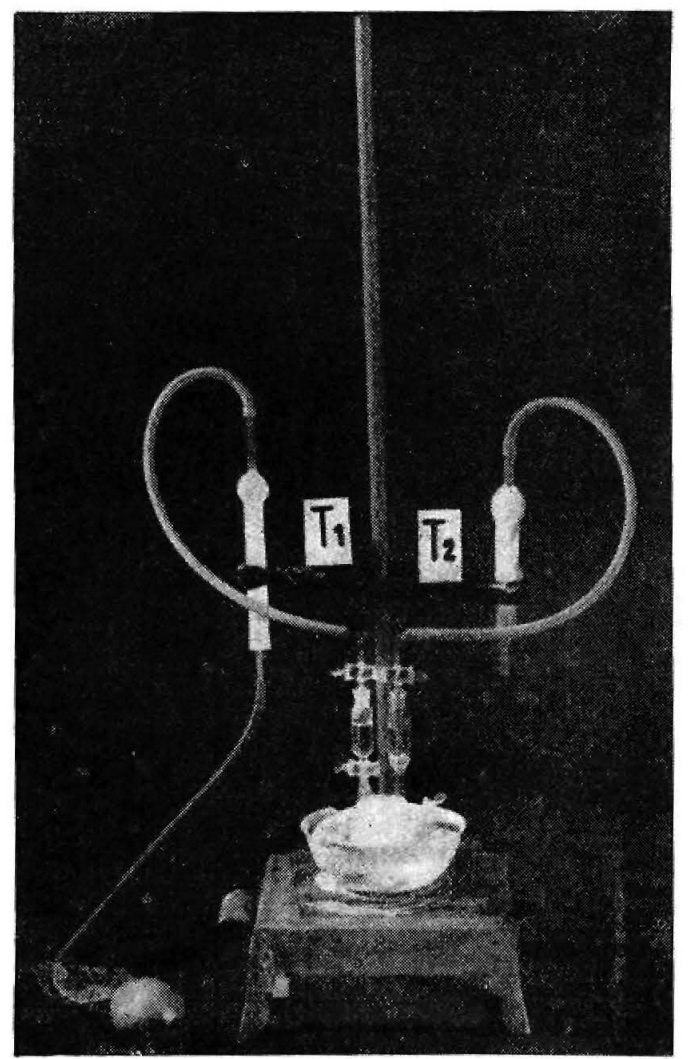

Fig. 1 


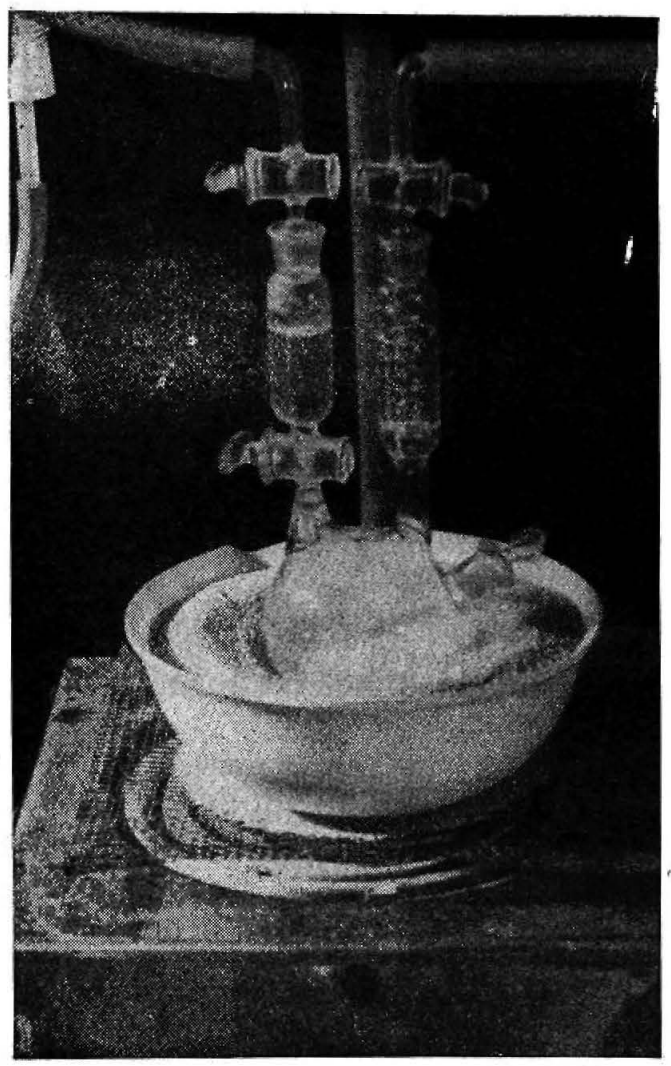

Fig. 2

llave $A_{1}$ y $A_{2}$, se cierran las llaves y se deja el conjunto en la caja de la balanza durante media hora.

Al cabo de este tiempo se abren durante un instante las llaves $A_{1}$ y $A_{2}$, se vuelven a cerrar y se pesa el matraz, utilizando otro análogo como tara. Si la balanza analítica de que se dispone es de un solo brazo, no cabe aplicar la medida de emplear como tara otro matraz. Se pesa aparte, y de manera aproximada, una cantidad de material previamente desecado a $120^{\circ} \mathrm{C}$ hasta peso constante ${ }^{(2)}$ (caliza, marga, o crudo de cemento portland) muy poco superior a la necesaria para la determinación (medio gramo, en general). Se introduce dicho material en el matraz por la boca F y, volviendo a cerrar ésta, se pesa de nuevo en las mismas condiciones anteriores. La diferencia entre esta pesada y la precedente del matraz corresponde a la cantidad de muestra utilizada en el ensayo.

Una vez asegurados todos los ajustes, se abren las llaves $A_{1}$ y $A_{2}$ y a continuación la $C$, haciendo que pase el ácido clorhídrico de $B_{1}$ al matraz.

Se produce entonces el ataque de la muestra y comienza el desprendimiento de $\mathrm{CO}_{2}$. El gas que sale por el tubo I (la llave C se encuentra ya cerrada) se ve obligado por la campana $G$ a bajar y pasar por los orificios $\mathrm{H}$, borboteando a través del ácido sulfúrico de $B_{2 .}$ y saliendo finalmente por $A_{2}$. Los ensanchamientos del tubo I y la propia campana $G$ sirven para retener las eventuales partículas líquidas arrastradas por los gases. El resto, así como la humedad de éstos, es retenido por el ácido sulfúrico, de forma que por $A_{2}$ sólo escapa el anhídrido carbónico.

Una vez producido el desprendimiento del $\mathrm{CO}_{2}$ en frio, se calienta suavemente el matraz en el baño de arena hasta completar el ataque de la muestra, ayudando con ello a la eliminación del gas. Es preferible el baño de arena al baño maría, pues con el primero se calienta en seco y el matraz no condensa humedad en su parte exterior.

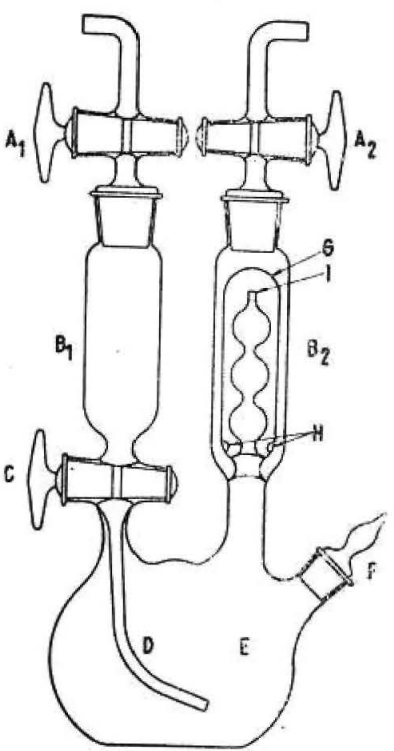

Fig. 3 
Se retira el matraz del baño y se deja enfriar. Una vez frío se conectan los tubos $T_{1}$ y $T_{g}$ que contienen cloruro cálcico anhidro, a las llaves $A_{1}$ y $A_{2}$, mediante uniones con tubos de goma estancos. Entonces, mediante vacio, o con una pera de goma, se hace pasar aire seco a través del matraz, previa apertura de las llaves $A_{1}, C y A_{g}$, con lo cual se expulsan los posibles restos de $\mathrm{CO}_{2}$.

Se guarda el matraz en un desecador, se deja después en la caja de la batanza durante media hora y se vuelve a pesar como al principio. La diferencia de esta pesada con la anterior es el peso del anhídrido carbónico desprendido, contenido en el peso de la muestra utilizada.

\section{RESULTADOS}

Con objeto de comprobar la eficacia del aparato y del método operatorio, se llevó a cabo una serje de determinaciones de $\mathrm{CO}_{z}$ con un material cuyo contenido de anhídrido carbónico es conocido. Se utilizó para ello un carbonato cálcico precipitado, con calidad de reactivo analítico, cuyo peso molecular y composición en tanto por ciento se admite son 100,09 , y $56,03 \% \mathrm{CaO}$ y $43,97 \% \mathrm{CO}_{2}$, respectivamente.

Se tomaron diferentes pesos de muestra pero, en general, se utilizaron cantidades del orden de medio gramo (2). Las determinaciones fueron 10 , y en 5 de ellas, por vía de comprobación, se halló el contenido de óxido cálcico, según (2). En estos casos se calcularon también las relaciones $\mathrm{CaO} / \mathrm{CO}_{2}$ a fin de compararlas con el valor teórico de 1,2743 , correspondiente al $\mathrm{CaCO}_{3} 100 \%$; igualmente se halló la suma de $\mathrm{CaO} \%+$ $\mathrm{CO}_{2} \%$, a fin de establecer el contenido total por ciento de $\mathrm{CaCO}_{3}$.

Los resultados están indicados en el cuadro 1, en el que se expresan también los porcentajes de $\mathrm{CO}_{2}$ calculados a partir de los de $\mathrm{CaO}$ hallados.

\begin{tabular}{|c|c|c|c|c|c|c|}
\hline \multirow{2}{*}{$\begin{array}{c}\text { Muestra } \\
\mathrm{N} .{ }^{\circ}\end{array}$} & \multirow{2}{*}{$\begin{array}{l}\text { Peso } \\
\mathrm{g}\end{array}$} & \multirow{2}{*}{$\begin{array}{c}\text { CaO hallado } \\
\%\end{array}$} & \multicolumn{2}{|c|}{$\mathrm{CO} \%$} & \multirow{2}{*}{$\begin{array}{c}\mathrm{CoO} / \mathrm{CO}_{2} \\
\text { teórico: } 1,2743\end{array}$} & \multirow{2}{*}{$\begin{array}{l}\text { Total } \% \\
\mathrm{CaCO}_{3}\end{array}$} \\
\hline & & & $\begin{array}{l}\text { Calculado } \\
\text { del CaO }\end{array}$ & Hallado & & \\
\hline 1 & 0,2393 & 55,71 & 43,72 & 43,67 & 1,2757 & 99,38 \\
\hline 2 & 0,4692 & 55,36 & 43,44 & 43,44 & 1,2744 & 98,80 \\
\hline 3 & 0,5258 & 55,34 & 43,43 & 43,40 & 1,2751 & 98,74 \\
\hline 4 & 0,5263 & - & - & 43,55 & - & - \\
\hline 5 & 0,5269 & 55,67 & 43,69 & 43,63 & 1,2759 & 99,30 \\
\hline 6 & 0,5270 & - & - & 43,25 & - & - \\
\hline 7 & 0,5367 & 55,45 & 43,51 & 43,47 & 1,2755 & 98,92 \\
\hline 8 & 0,5457 & - & - & 43,27 & - & - \\
\hline 9 & 0,5459 & - & - & 43,30 & - & - \\
\hline 10 & 0,6024 & - & - & 43,66 & - & - \\
\hline $\begin{array}{l}\text { Valores } \\
\text { medios }\end{array}$ & - & 55,51 & 43,56 & 43,46 & 1,2753 & 99,03 \\
\hline
\end{tabular}

Como puede apreciarse, los valores del $\mathrm{CO}_{2} \%$ hallados, y los calculados a partir del $\mathrm{CaO} \%$ concuerdan bastante bien y, por consiguiente, también concuerdan bastante bien las relaciones $\mathrm{CaO} / \mathrm{CO}_{2}$ con respecto al valor teórico de 1,2743. Pese a lo cual, los valores de $\mathrm{CaO}$ y los de $\mathrm{CO}_{2}$ son algo inferiores o los teóricos, por lo que los de $\mathrm{CaCO}_{3}$ no llegan a $100 \%$. La diferencia, que en la cifra media es de $0,97 \%$, parece 
indicar que el carbonato cólcico utilizado tiene, en la citada cuantía, alguna impureza no determinada.

En vista de ello se efectuó otra serie de 15 ensayos para deferminar la pérdida al fuego del producto a $1.000^{\circ} \mathrm{C}$. Los resultados quedan indicados en el cuadro 2. Se observa que, tanto los valores individuales como el valor medio, son más altos que el teórico, lo cual, unido a los resultados del cuadro 1, hace pensar en que la diferencia media de $0,97 \%$ hallada en aquél es debida a una impureza de tipo volátil entre $120^{\circ} \mathrm{C}$ (temperatura de desecación de la muestra) y $1.000^{\circ} \mathrm{C}$ (temperatura de calcinación en la determinación de la pérdida al fuego). Al no acusarse dicha diferencia por el procedimiento descrito para la determinación del $\mathrm{CO}_{z}$, la conclusión provisional es que se debe a agua unida al carbonato cálcico en una forma tal que no se desprende a $120^{\circ} \mathrm{C}$.

\section{CUADRO 2}

$\begin{array}{ccc}\text { Muestra } & \text { Peso } & \text { Pérdida al fuego } \\ \text { N. } & g & \% \\ 1 & 0,2519 & 44,10 \\ 2 & 0,2553 & 43,99 \\ 3 & 0,2565 & 44,21 \\ 4 & 0,5003 & 44,21 \\ 5 & 0,5005 & 44,18 \\ 6 & 0,5005 & 44,12 \\ 7 & 0,5006 & 44,13 \\ 8 & 0,5008 & 44,31 \\ 9 & 0,5016 & 44,14 \\ 10 & 0,5023 & 44,26 \\ 11 & 0,5029 & 44,06 \\ 12 & 0,5033 & 44,13 \\ 13 & 0,5050 & 44,30 \\ 14 & 0,5054 & 44,00 \\ 15 & 0,5068 & 44,28 \\ & & \\ \text { Valor } & - & 44,16 \\ \text { medio } & & \end{array}$

Sentado esto, se procedió a corregir los pesos de las muestras que figuran en el cuadro $1, y$ también los valores de $\mathrm{CaO}$ y
$\mathrm{CO}_{2}$ hallados y el del $\mathrm{CO}_{2}$ calculado, asi como el de las relaciones $\mathrm{CaO} / \mathrm{CO}_{2}$ y el de los porcentajes totales de $\mathrm{CaCO}_{3}$.

En consecuencia, el cuadro 1 queda transformado en el cuadro 3.

Como es natural, los valores concuerdan con los teóricos.

De igual manera se corrigieron los pesos de las muestras que figuran en el cuadro 2 $y$, por lo tanto, los de la pérdida al fuego. El cuadro 2 queda entonces convertido en el cuadro 4, en el que, de la pérdida al fuego corregida, se desglosa el agua supuesta, quedando el resto como $\mathrm{CO}_{2}$. Puede observarse que, tanto los valores individuales como el valor medio de $\mathrm{CO}_{2}$ así deducidos, concuerdan mucho mejor con los valores del cuadro 3 , e incluso con los del cuadro 1.

Queda por demostrar, de una manera convincente, la existencia de ese $0,97 \%$ que se ha supuesto agua unida al carbonato cálcico. Para ello se introdujeron muestras del producto en un horno, a temperaturas de $200,300,400,500$ y $600^{\circ} \mathrm{C}$, y en estufa a 100 y $120^{\circ} \mathrm{C}$, hasta peso constante en cada caso.

En la correspondiente curva (A) de la figura 4, en la que se expresan los porcentajes de pérdida de peso en función de la temperatura, se observa que por encima de los $120^{\circ} \mathrm{C}$ y hasta los $550^{\circ} \mathrm{C}$ hay una pérdida de peso ligera pero creciente. Después hay una pérdida fuerte y rápida que corresponde a la descarbonatación. Esta tiene lugar, en condiciones normales, a $825^{\circ} \mathrm{C}$, pero dada la menor presión ambiente (unos $703 \mathrm{~mm}$ de mercuriol y la calefacción brusca en el horno, junto con la posible influencia de ese agua hipotética, presente como impureza, el comienzo de la descarbonatación se produce mucho antes de alcanzarse la citada temperatura.

Se determinó también la curva termogravimétrica (B) del producto, en una termobalanza tipo Chevenard, empleando I gramo de muestra y una velocidad de calefacción de $150^{\circ} \mathrm{C} /$ hora. Se registró gráfica y automáticamente la curva resultante, la cual, como la representativa de las pérdidas de peso en el horno, indica pérdidas ligeras y continuas, a partir de los $275^{\circ} \mathrm{C}$ y hasta los $600^{\circ} \mathrm{C}$. A esta temperatura aparece un punto de inflexión, a partir del cual 
comienza la descarbonatación. Esta se produce después que en el caso del horno, sin duda por ser más suove y lenta la calefacción, lo que también explica las menores pérdidas observadas en la primera rama de la curva, a igualdad de temperatura.

El punto de inflexión corresponde a una pérdida de peso que, expresada como tanto por ciento del peso de la muestra empleada $(1,0000 \mathrm{~g})$, viene a ser de un $1 \%$, lo cual está de acuerdo con el $0,97 \%$ deducido y supuesto como agua. No puede ser otra cosa, pues el carbonato cálcico utilizado no contiene otras materias volátiles, ni orgánicas ni inorgánicas, que de manera lenta y continua puedan desprenderse en los intervalos de temperatura señalados. Este agua va incluida en la pérdida al fuego total determinada a $1.000^{\circ} \mathrm{C}$, pero no en la determinación del $\mathrm{CO}_{3}$ por el método descrito. De aquí que los valores del $\mathrm{CO}_{2}$ hallados por este método concuerden con los de $\mathrm{CaO}$ determinados analíticamente y no con los de la pérdida al fuego, a no ser los corregidos.

\section{CONCLUSIONES}

El aparato y procedimiento descritos para la determinación de $\mathrm{CO}_{2}$ es aplicable a aquellos productos o materiales que no contengan otras materias volátiles, excepción hecha del agua, que no interfiere. Es aplicable a calizas puras $y$, eventualmente, a margas, crudos para cemento, yesos, etc.
El método es más cómodo, sencillo y rápido que los volumétricos y gravimétricos por absorción, y su precisión es suficiente para lo que se exige en los casos de aplicación práctica. Concretamente lo demuestra el hecho casual de haberlo ensayado con un producto que se creía puro y contenía un $1 \%$ aproximadamente de agua, lo cual fué puesto de manifiesto por el método y comprobado después por otras técnicas de mayor precisión.

\section{BIBLIOGRAFIA}

1. DAVIS, A. C.: Portland Cement, pógina 57. Londres, 1943.

2. Normas de Ensayo del Laboratorio Central. NELC 3.02-a.

3. Normas de Ensayo del Laboratorio Central. NELC 3.03-a; A. S. T. M. C 26-42.

4. VILLAVECCHIA, V:: Químico Analítica Aplicada, tomo I, pág. 307. Barcelona, 1949.

5. BERL - LUNGE - D'ANS: Métodos de Anólisis Químico-Industrial, tomo I, pág. 638. Madrid, 1946.

6. GASPAR, D.: Arcillas, pág. 170. Instituto Técnico de la Construcción y del Cemento. Madrid, 1951. 
CUADRO 3

\begin{tabular}{|c|c|c|c|c|c|c|}
\hline \multirow{2}{*}{$\begin{array}{c}\text { Muestra } \\
\mathrm{N}^{\circ}\end{array}$} & \multirow{2}{*}{$\begin{array}{c}\text { Peso corregido } \\
\mathrm{g}\end{array}$} & \multirow{2}{*}{$\begin{array}{c}\text { CaO hallado } \\
\text { (corregido) } \\
\%\end{array}$} & \multicolumn{2}{|c|}{$\mathrm{CO}_{z} \%$ corregido } & \multirow{2}{*}{$\begin{array}{c}\mathrm{CaO} / \mathrm{CO}: \\
\text { (corregido) } \\
\text { teórico: } 1,2743\end{array}$} & \multirow{2}{*}{$\begin{array}{l}\text { Total \% } \\
\text { kcorregido }\end{array}$} \\
\hline & & & $\begin{array}{l}\text { Calculado } \\
\text { del } \mathrm{CaO}\end{array}$ & Hallado & & \\
\hline 1 & 0,2370 & 56,25 & 44,14 & 44,09 & 1,2758 & 100,34 \\
\hline 2 & 0,4646 & 55,91 & 43,87 & 43,87 & 1,2744 & 99,78 \\
\hline 3 & 0,5207 & 55,88 & 43,86 & 43,82 & 1,2752 & 99,70 \\
\hline 4 & 0,5212 & - & - & 43,98 & - & - \\
\hline 5 & 0,5218 & 56,21 & 44,12 & 44,06 & 1,2758 & 100,27 \\
\hline 6 & 0,5219 & - & - & 43,67 & - & - \\
\hline 7 & 0,5317 & 55,97 & 43,92 & 43,88 & 1,2755 & 99,87 \\
\hline 8 & 0,5404 & - & - & 43,69 & - & - \\
\hline 9 & 0,5406 & - & - & 43,72 & - & - \\
\hline 10 & 0,5966 & - & - & 44,08 & - & - \\
\hline $\begin{array}{l}\text { Valores } \\
\text { medios }\end{array}$ & - & 56,04 & 43,98 & 43,89 & 1,2753 & 99,99 \\
\hline
\end{tabular}

CUADRO 4

$\begin{array}{ccccc}\begin{array}{c}\text { Muestra } \\ \text { N. }\end{array} & \text { Peso corregido } & \begin{array}{c}\text { Pérdida al fuego } \\ \%\end{array} & \begin{array}{c}\text { Aguo supuesta } \\ \text { corregido }\end{array} & \begin{array}{c}\text { CO } \% \\ \text { calculado }\end{array} \\ 1 & g & 44,54 & 0,97 & \\ 2 & 0,2494 & 44,42 & 0,97 & 43,57 \\ 3 & 0,2528 & 44,57 & 0,97 & 43,45 \\ 4 & 0,2544 & 44,65 & 0,97 & 43,58 \\ 5 & 0,4954 & 44,62 & 0,97 & 43,68 \\ 6 & 0,4956 & 44,56 & 0,97 & 43,59 \\ 7 & 0,4956 & 44,57 & 0,97 & 43,60 \\ 8 & 0,4957 & 44,75 & 0,97 & 43,78 \\ 9 & 0,4959 & 44,58 & 0,97 & 43,61 \\ 10 & 0,4967 & 44,70 & 0,97 & 43,73 \\ 11 & 0,4974 & 44,49 & 0,97 & 43,52 \\ 12 & 0,4980 & 44,56 & 0,97 & 43,59 \\ 13 & 0,4984 & 44,73 & 0,97 & 43,76 \\ 14 & 0,5001 & 44,43 & 0,97 & 43,46 \\ 15 & 0,5005 & 44,71 & 0,97 & 43,74 \\ & 0,5019 & & & \\ \text { Valores } & & 44,69 & 0,97 & 43,63 \\ \text { medios } & - & & & \end{array}$




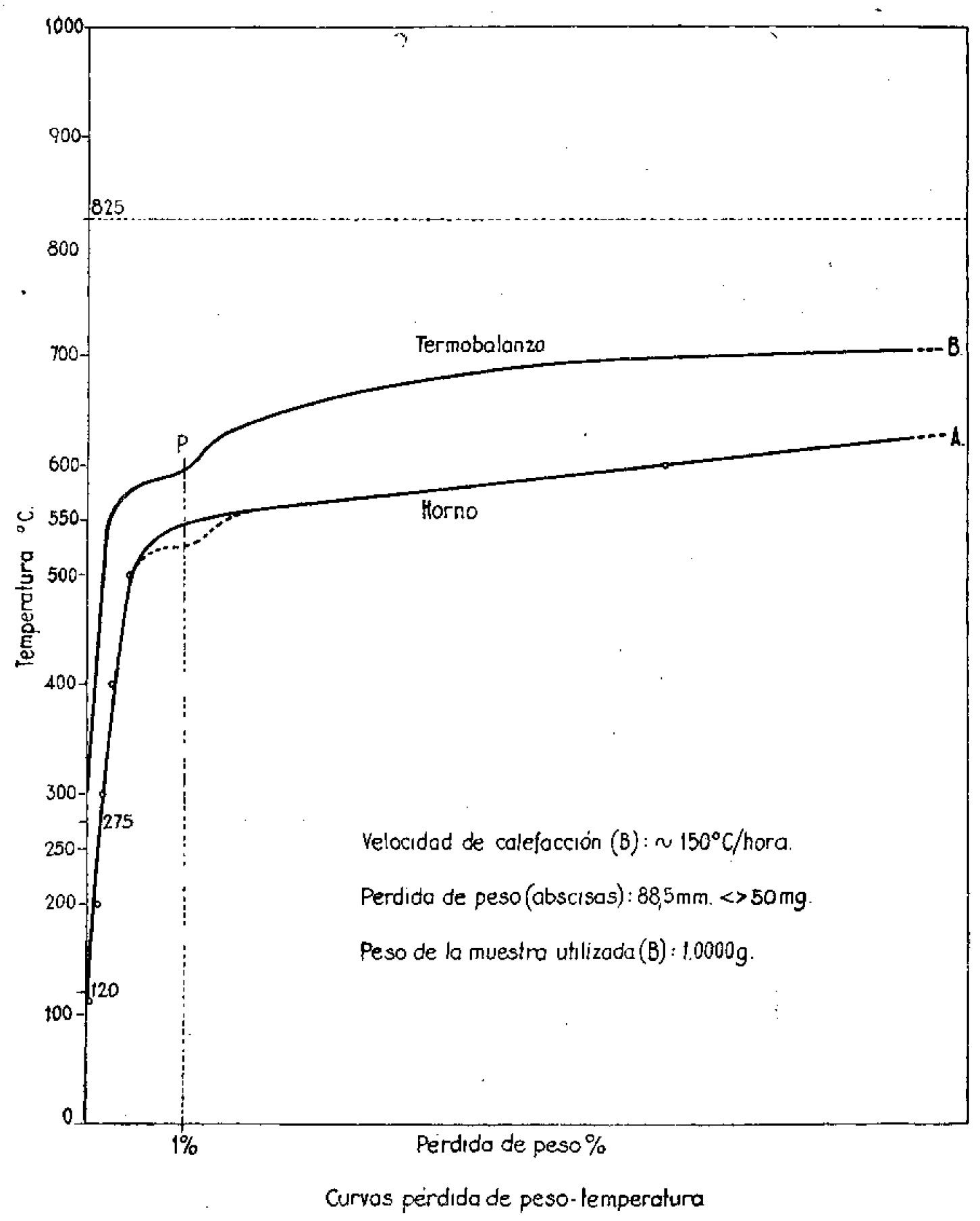

Fig. 4 